\title{
Qualidade de vida e instrumentos de avaliação em saúde materna
}

\author{
Rosalinda Asenjo López Berti*, Lislaine Aparecida Fracolli, D.Sc.**, Danielle Freitas Alvim de Castro***
}

*Enfermeira, Mestranda em Ciências pela Escola de Enfermagem da Universidade de São Paulo, Docente da Escola Técnica de Saúde da Universidade Federal de Uberlândia, Membro do grupo de pesquisa Modelos Tecno-Assistênciais do Departamento de Saúde Coletiva da Escola de Enfermagem da USP, Membro ativo da ONG "Missão África", **Enfermeira, Prof. Departamento de Saúde Coletiva da Escola de Enfermagem da Universidade de São Paulo, Coordenadora do grupo de pesquisa Modelos TecnoAssistênciais do departamento de Saúde Coletiva da Escola de Enfermagem da USP, ${ }^{* * *}$ Doutoranda em Ciências pela Escola de Enfermagem da Universidade de São Paulo, Membro do grupo de pesquisa Modelos Tecno-Assistênciais do departamento de Saúde Coletiva da Escola de Enfermagem da USP

\section{Resumo}

A qualidade de vida é um construto que vem assumindo importância relevante nos últimos anos, concomitantemente existe um crescimento no número de instrumentos para mensurar a qualidade de vida $(\mathrm{QV})$. Durante a gestaçáo, alguns instrumentos de QV são utilizados no período de pré-natal e pós-parto, dentre eles instrumentos genéricos ou instrumentos específicos, em diferentes países e de forma esporádica. Foi realizado um levantamento bibliográfico de artigos científicos nacionais e internacionais para esclarecer de maneira coerente o assunto em questão. Assim, 140 artigos foram selecionados, destes somente 10 artigos responderam à questáo central da pesquisa. As categorias que emergiram foram: A qualidade de vida da gestante e os sintomas de náuseas e vômitos, violência sexual, sintomas de depressão, e adaptação à gravidez e a percepçáo da gestante de modo geral de sua qualidade de vida. Concluímos que este estudo pode contribuir no estimulo da utilização de instrumentos de qualidade de vida em gestantes, na aquisição de subsídios reais, para implantação de programas, estratégias de atendimento e intervenção adequada à realidade das gestantes.

Palavras-chave: qualidade de vida, gravidez, avaliação de programas e instrumentos de pesquisa, pré-natal, pós-natal.

\section{Abstract \\ Quality of life and tools of assessment in maternal health}

Quality of life is a construct that has assumed great importance in recent years, there is a concomitant increase in the number of instruments to measure quality of life (QL). During pregnancy some instruments of QL are used in the prenatal and post-partum, among them we have generic or specific instruments, in different countries and sporadically. We conducted a literature review of national and international scientific articles aiming at to clarifying the issue at hand. Thus, a total of 140 articles were selected, but only 10 answered to the central research question. The categories that emerged were: the quality of life of the pregnant woman and the symptoms of nausea and vomiting, sexual violence, 
symptoms of depression, adaptation to pregnancy and the perception of the pregnant woman regarding her quality of life in general. We concluded that this study may contribute to stimulate the use of instruments for quality of life in pregnant women, to acquire subsidies, for implementation of programs and appropriate care strategies and intervention to the reality of pregnant women.

Key-words: quality of life, pregnancy, evaluation of research programs and tools, prenatal, postnatal.

\section{Resumen}

\section{Calidad de vida y los instrumentos de evaluación en salud materna}

La calidad de vida es un constructo que ha adquirido gran importancia en los últimos ańos, hay un aumento concomitante en el número de instrumentos para medir la calidad de vida (CV). Durante el embarazo algunos instrumentos de CV fueron usados durante el período prenatal y postparto, entre ellos instrumentos genéricos y específicos, en diferentes países y de forma esporádica. Se realizó una revisión bibliográfica de artículos científicos nacionales e internacionales para aclarar el tema en cuestión. Así, se seleccionaron un total de 140 artículos, pero sólo 10 respondieron a la pregunta central de la investigación. Las categorías que surgieron fueron: La calidad de vida de la mujer embarazada y los síntomas de náuseas y vómitos, la violencia sexual, los síntomas de la depresión, la adaptación al embarazo y la calidad de vida, en general, percibida por la mujer embarazada. Se concluye que este estudio puede contribuir a estimular el uso de instrumentos de calidad de vida en mujeres embarazadas, en la adquisición de subsidios reales, para la implementación de programas y estrategias de atención e intervención adecuada a mujeres embarazadas.

Palabras-clave: calidad de vida, embarazo, evaluación de programas e instrumentos de investigación, prenatal, postnatal.

\section{Introdução}

A gravidez é um período de profundas transformações e, embora seja um processo fisiológico, cada mulher reage às mudanças advindas desse processo de forma particular. Nesse período fatores como história pregressa, o desejo ou não da gravidez tem influencia em sua qualidade de vida (QV).

Considera-se que as condiçóes sociais, econômicas e demográficas, bem como fatores biológicos, obstétricos e patológicos interferem no desenvolvimento de uma gestação saudável. Essas gestantes precisam ser preparadas para a maternidade, desde o início da gestação, recebendo orientaçóes e esclarecendo dúvidas, principalmente quanto ao nascimento, objetivando compreender o momento do parto como um processo natural, capaz de superar o medo da dor e alcançar a realizaçáo da maternidade [1].

Discutir fatores que afetam a saúde da gestante significa valorizar parâmetros ampliados de cuidado em saúde da mulher, além do controle de sintomas e necessidades durante a gestação. Além disso, faz-se importante definir problemas e lacunas do serviço, implantar estratégias efetivas para a $\mathrm{QV}$ da gestante e estabelecer prioridades.

Ao analisar as necessidades das gestantes e sua percepçáo em quanto ao bem estar durante este período, Souza [2] afirma que "para satisfazer a gestante, é imprescindível conhecer suas necessidades, expectativas e percepçóes".

Um conceito que tem abrangido de maneira ampliada o construto qualidade de vida é o conceito da Organização Mundial da Saúde (OMS) [3], que criou um grupo para desenvolver a medição da QV, propondo a seguinte definição: "A Qualidade de Vida é a percepção do indivíduo da sua posição na vida, no contexto da sua cultura e no sistema de valores em que vive e em relaçáo a suas expectativas, seus padróes e suas preocupaçôes”.

Este conceito abrange a complexidade do construto e inter-relaciona o meio ambiente com aspectos físicos, psicológicos, nível de independência, relaçóes sociais e crenças pessoais. Desse modo, entrelaça três aspectos fundamentais:

Subjetividade: a perspectiva do indivíduo é o que está em questão. A realidade objetiva só conta à medida que é percebida pelo indivíduo.

Multidimensionalidade: a qualidade de vida é composta por várias dimensôes - física, mental, social, etc.

Presença de dimensóes positivas e negativas: para uma ótima $Q V$, é necessário que estejam presentes alguns elementos, por exemplo, a mobilidade, e outros se façam ausentes, por exemplo, a dor [4]. 
Portanto, a qualidade de vida é um conceito amplo que envolve de maneira complexa a saúde física, o estado psicológico, o nível de independência, as relaçốes sociais as crenças pessoais dos indivíduos e suas relaçóes com características predominantes [3].

$\mathrm{Na}$ atualidade existem duas formas de mensurar a qualidade de vida por meio de instrumentos genéricos e instrumentos específicos. Os genéricos são utilizados na avaliação de qualidade de vida da população em geral, sendo mais apropriado a estudos epidemiológicos, planejamento e avaliaçáo do sistema de saúde. Os mais utilizados no mundo são: The Medical Outcomes Study 36 - Item Short Form Health Survey (SF-36) e o WHOQOL-100 da OMS [3-5]. Já os instrumentos específicos são capazes de avaliar, de forma individual e específica, determinados aspectos da qualidade de vida.

$\mathrm{O}$ interesse crescente pela avaliação da QV e os instrumentos para mensurá-la é, em parte, decorrente de novos conceitos de saúde que foram elaborados por conferências Internacionais de Promoção da Saúde, constituindo um novo modo de ver a saúde e a doença, rompendo a hegemonia do modelo biomédico $[4,5]$.

A utilização de instrumentos de qualidade de vida em gestantes se faz necessária para a implantação de programas e estratégias de atendimento e intervenção adequadas à realidade das gestantes, uma vez que estes instrumentos de avaliação fornecem subsídios que auxiliam na avaliaçáo ampliada do período gestacional [6].

Tendo em vista as potencialidades que a avaliaçáo de QV no período gestacional possa ter, este estudo teve como finalidade levantar na literatura trabalhos que fizeram uso de instrumentos de avaliação de QV neste período.

\section{Material e métodos}

O presente estudo utilizou o método da pesquisa bibliográfica de natureza qualitativa baseada na metodologia da revisão da literatura sobre instrumentos de avaliação de qualidade de vida em gestantes [6]. A pesquisa realizada usou a estratégia de levantamento bibliográfico de artigos publicados com o auxílio do operador booleano "and" com os seguintes termos de busca: "qualidade de vida", "avaliação de programas e instrumentos de pesquisa", "pré-natal" e "pós-natal" por meio de busca eletrônica nos bancos de dados, Pubmed, Psycoinfo,
Dedalus e Cinahal. Nesta pesquisa, o período compreendeu a literatura publicada entre 2008 e 2011.

Figura 1 - Fases da seleção dos dados.

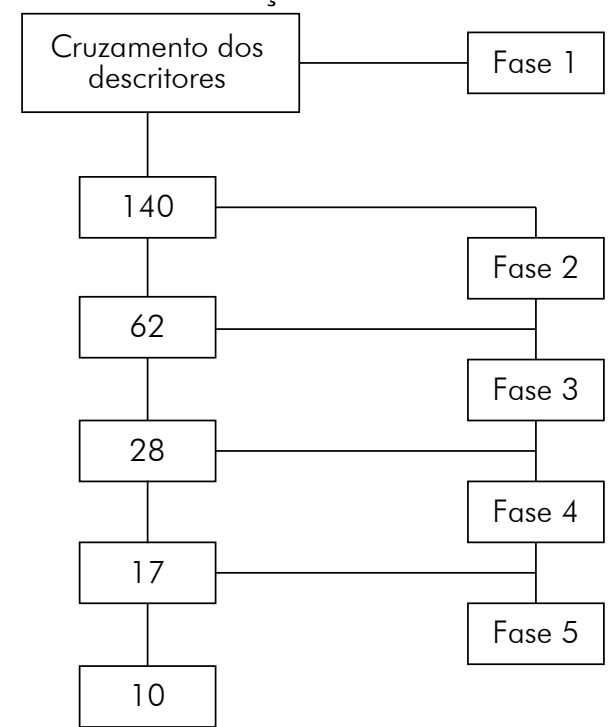

Fonte: Berti RAL. Diagrama do cruzamento de descritores relacionados a instrumentos de avaliação de qualidade de vida em gestantes. São Paulo: EEUSP; 2013.

Para a busca foram selecionados 140 artigos, lidos 17 artigos, no entanto apenas 10 que responderam à questão central da revisão foram utilizados. Critérios considerados para a inclusão do artigo foram os seguintes: artigos que fossem publicados em português e inglês. Artigos pertinentes ao tema proposto que contemplassem os objetivos deste estudo. Foram excluídos artigos em outros idiomas, relatos de casos e artigos que não eram pertinentes ao tema.

\section{Resultados}

$\mathrm{Na}$ Figura 2 apresentamos os tipos de instrumentos que foram utilizados durante a gravidez: entre os instrumentos genéricos foram utilizados o WHOQOL - Breaf, e o MOS-SF36 e entre os instrumentos de qualidade de vida específicos em gestantes foram: Health Related Quality of Life for Nausea and Vomiting (NVPQol), Questionário de Qualidade de Vida no Pré-Natal, Multicultural Quality OfLife Índex (MQLI) questionnaires, Índice de bem estar Australia Unity e Qualidade de Vida Europeu (EuroQol 6D 3L).

Apesar da existência de uma preocupação em relação à maternidade e seus cuidados, poucas são as pesquisas relacionadas à qualidade de vida da gestante em seu contexto social, cultural e psicológico. 
Figura 2 - Tipos de instrumentos utilizados para avaliar gestantes.

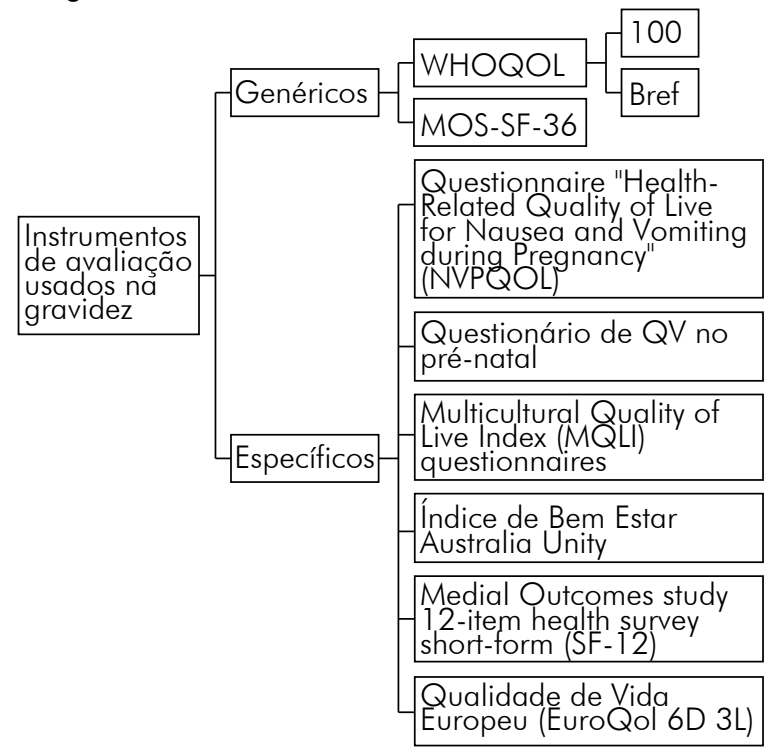

Fonte: Berti RAL. Diagrama de instrumentos genéricos e específicos de avaliação utilizados na gravidez: revisão integrativa da leitura. São Paulo: EEUSP; 2013.

O Quadro 1 descreve os instrumentos de qualidade de vida utilizados em gestantes de diferentes países, apresentando referencia dos autores, tipos de instrumentos de qualidade de vida utilizados no período da gravidez, e os anos em que foram publicados. Desse total de publicaçóes selecionadas encontramos: uma publicaçáo em 2008, cinco publicaçôes em 2009, duas publicaçôes em 2010 e duas em 2011.

Esses instrumentos abordaram diferentes focos temáticos dentro período gestacional da mulher visando avaliar à qualidade de vida. Entre os temas abordados estão: A adaptação da gestante durante o período pré-natal, a violência sexual, a validaçáo do instrumento WHOQOL-bref entre as mulheres após parto, validação do instrumento específico para sintomas de náuseas e vômitos, qualidade de vida em gestantes com retardo uterino, qualidade de vida em gestantes atendidas em uma maternidade social, gestantes assistidas pela estratégia saúde da família e a qualidade de vida em gestantes que apresentam sintomas depressivos.

A violência sexual foi avaliada com os instrumentos Medical Outcomes Study 12-item health survey short-form (SF-12-) + questionário sobre violência e dados demográficos e sua associação com autopercepçáo de saúde entre mulheres grávidas, analisando que o histórico de violência sexual durante a gestação apresenta alta prevalência de $39,1 \%$, diminuindo a autopercepçáo de saúde [7].
A violência durante a gestação, de acordo com a pesquisa, provoca efeitos psicológicos negativos sendo apontado como agressor o parceiro. Estudos mostraram também que as gestantes que sofreram stress por violência apresentam experiências dissociativas e amnésia das vivências traumáticas associado à incapacidade das vítimas de elaborar e sair de situações de violência doméstica [8].

Para a pesquisa que avaliou os sintomas mais comuns de náuseas e vômitos, durante a gestaçáo, foi utilizado o instrumento "Health-Related Quality of Life for Nausea and Vomiting during Pregnancy" (NVPQOL), identificando que o impacto de náuseas e vômitos influencia de forma considerável a capacidade de executar atividades diárias habituais, afetando a vida familiar, o funcionamento social e elevando os níveis de estresse. Além disso, prejudica o sono e o desempenho no trabalho e incapacitando a mulher de suas atividades normais [9].

Segundo Bijlenga et al. [10], gestantes com retardo de crescimento uterino (CIUR), considerado uma gravidez de alto risco, em que é indicada a induçáo do parto, foram utilizados os instrumentos Short-Form ( FS-36) + qualidade de vida europeu (EuroQol 6D 3L)+ escala de depressão (HADS), concluindo que esse tipo de cirurgia em gestantes não afetou de forma considerável a $\mathrm{QV}$ da gestante.

No Brasil, a avaliação da qualidade de vida em gestantes assistidas pela Estratégia Saúde da Família foi realizada por Castro [11], que analisou as potencialidades do instrumento WHOQOL- bref, durante o pré-natal, na qual a pesquisadora sugere que este instrumento poderia ser empregado como tecnologia de monitoramento da clínica ampliada na assistência pré-natal a gestantes.

$\mathrm{O}$ instrumento de qualidade de vida em gestante mais utilizado foi o WHOQOL- bref, em conjunto com outros instrumentos conforme mostra o Quadro 1 acima citado. Esse instrumento foi criado pelo grupo de qualidade de vida da OMS [3]. E o instrumento de qualidade de vida Medical Outcomes Study (MOS-SF36) ficou em segundo lugar como instrumento mais utilizado em gestantes.

Segundo Lima e Webster [12-13] que utilizaram os instrumentos como o (HRQol)+ (MOS-SF 36) Whoqol-breaf+ índice de depressão pós-parto de Edimburgo+ Índice de Bem Estar Australiana Unity. A depressão pós-parto é um distúrbio do humor de grau moderado e severo sendo detectada entre a sexta e oitava semana pós-parto influenciando de forma negativa a qualidade de vida durante o puerpério da mulher. 
Quadro 1 - Sumarização das publicações selecionadas.

\begin{tabular}{|c|c|c|c|}
\hline $\begin{array}{l}\text { Portais e bases de } \\
\text { dados }\end{array}$ & $\begin{array}{c}\text { Assuntos abordados: } \\
\text { Gestantes e QV }\end{array}$ & Instrumentos de QV & Autores /Publicações \\
\hline \multirow[t]{4}{*}{ Pubmed } & $\begin{array}{l}\text { Development of a Chinese } \\
\text { short form of the prenatal } \\
\text { self-evaluation Questionnai- } \\
\text { re. }\end{array}$ & $\begin{array}{l}\text { Questionário de autoavaliação } \\
\text { do pré-natal para adaptação a } \\
\text { gravidez visando a QV. }\end{array}$ & $\begin{array}{l}\text { Lin CT, Cheng CP, Kuo } \\
\text { SH, Chou FH. J Clin Nurs } \\
2009 ; 18(5): 659-66 .\end{array}$ \\
\hline & $\begin{array}{l}\text { An assessment of maternal } \\
\text { quality of life in the postpar- } \\
\text { tum period in southern Brazil: } \\
\text { a comparison of the two } \\
\text { questionnaires. }\end{array}$ & $\begin{array}{l}\text { WHOQOL-Bref and Multicultu- } \\
\text { ral Quality of Life Index (MQLI) } \\
\text { questionnaires }\end{array}$ & $\begin{array}{l}\text { Zubaran C, Foresti K, Schu- } \\
\text { macher MV, Muller LC, Amo- } \\
\text { retti AL. Clinics (São Paulo) } \\
\text { 2009;64(8):751-6. }\end{array}$ \\
\hline & $\begin{array}{l}\text { Sexual violence and its } \\
\text { association with health self- } \\
\text { perception among pregnant } \\
\text { women. }\end{array}$ & 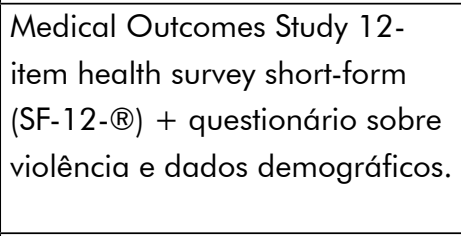 & $\begin{array}{l}\text { Aquino NM, Sun SY, Olivei- } \\
\text { ra EM, Martins MG, Silva JF, } \\
\text { Mattar R. } \\
\text { Rev Saúde Pública } \\
2009 ; 43(6): 954-60 .\end{array}$ \\
\hline & $\begin{array}{l}\text { Validation of the WHOQOL- } \\
\text {-breaf among women follo- } \\
\text { wing childbirth }\end{array}$ & $\begin{array}{l}\text { Whoqol-breaf+índice de de- } \\
\text { pressão pós-parto de Edim- } \\
\text { burgo+ Índice de Bem Estar } \\
\text { Australiana Unity. }\end{array}$ & $\begin{array}{l}\text { Webster J, Nicholas C, Velacott } \\
\text { C, Cridland N, Fawcett L. } \\
\text { Aust N Z J Obstet Gynaecol } \\
2010 ; 50(2): 132-7 .\end{array}$ \\
\hline \multirow[t]{2}{*}{ Psycoinfo } & $\begin{array}{l}\text { Validation of the nausea } \\
\text { and vomiting of pregnancy } \\
\text { specific health related quality } \\
\text { of life questionnaire. }\end{array}$ & $\begin{array}{l}\text { questionnaire "Health-Related } \\
\text { Quality of Life for Nausea and } \\
\text { Vomiting during Pregnancy" } \\
\text { (NVPQOL) }\end{array}$ & $\begin{array}{l}\text { Lacasse and Bérard } 2008 . \\
\text { Research Center, CHU Sainte- } \\
\text { - Justine, Montreal, Quebec, } \\
\text { Canada. } \\
\text { Periódics. Quality of life rese- } \\
\text { arch }\end{array}$ \\
\hline & $\begin{array}{l}\text { Maternal health-related qua- } \\
\text { lity of life after induction of } \\
\text { labor or expectant monitoring } \\
\text { in pregnancy complicated by } \\
\text { intrauterine growth retarda- } \\
\text { tion beyond } 36 \text { weeks. }\end{array}$ & $\begin{array}{l}\text { Short-Form (FS-36) + qua- } \\
\text { lidade de vida europeu (Eu- } \\
\text { roQol 6D 3L+ escala de } \\
\text { depressão(HADS). }\end{array}$ & $\begin{array}{l}\text { Denice Bijlenga et al. } \\
\text { Department of social Medicine, } \\
\text { Academic medical Center. } \\
\text { Amsterdam, The Netherlands, } \\
2011 \text {. Periodics quality of life } \\
\text { research. }\end{array}$ \\
\hline \multirow[t]{4}{*}{ Dedalus } & $\begin{array}{l}\text { Qualidade de vida relacio- } \\
\text { nada à saúde da mulher } \\
\text { grávida: dados preliminares. }\end{array}$ & $\begin{array}{l}\text { Medical Outcomes Study (MOS- } \\
\text {-SF36) }\end{array}$ & $\begin{array}{l}\text { Dias CM. Trabalho de evento. } \\
\text { Resumo - Nacional. São Paulo: } \\
\text { Escola de Enfermagem da USP; } \\
2009 .\end{array}$ \\
\hline & $\begin{array}{l}\text { Qualidade de vida relacio- } \\
\text { nada à saúde de mulheres } \\
\text { gestantes atendidas em uma } \\
\text { maternidade social }\end{array}$ & $\begin{array}{l}\text { Medical Outcomes Study (MOS- } \\
\text {-SF36) }\end{array}$ & $\begin{array}{l}\text { Murata M. Trabalho de evento. } \\
\text { Resumo - Nacional. São Paulo: } \\
\text { Escola de Enfermagem da USP; } \\
2009 .\end{array}$ \\
\hline & $\begin{array}{l}\text { Qualidade de vida de ges- } \\
\text { tantes assistidas pela estraté- } \\
\text { gia saúde da família. }\end{array}$ & $\begin{array}{l}\text { WHOQOL Bref + questionário } \\
\text { sociodemográfico }\end{array}$ & $\begin{array}{l}\text { Castro DFA. Dissertação de } \\
\text { mestrado. São Paulo: Escola de } \\
\text { Enfermagem da USP; } 2010 .\end{array}$ \\
\hline & $\begin{array}{l}\text { QV relacionada à saúde } \\
\text { de mulheres grávidas com } \\
\text { sintomas depressivos }\end{array}$ & $\begin{array}{l}\text { Health related Qol of life (HR- } \\
\text { Qol)+ Medical outcomes study } \\
\text { 36-item short form health survey } \\
\text { (MOS-SF 36) }\end{array}$ & $\begin{array}{l}\text { Lima MOP. Tese de Doutorado. } \\
\text { São Paulo: Escola de Enferma- } \\
\text { gem da USP; } 2011\end{array}$ \\
\hline
\end{tabular}

Fonte: Portais e Banco de dados: Pubmed; Psycoinfo e Dedalus. São Paulo, 2012. 


\section{Conclusão}

A compreensão da QV no período gestacional leva-nos a ampliar nossa visão de mundo visando à integralidade, fornecendo subsídios para a intervenção dos cuidados neste processo gestacional em que ocorrem grandes mudanças tanto biológicas, físicas e psíquicas na mulher.

Acredita-se que as informaçóes geradas a partir dos instrumentos utilizados, servem como indicadores que avaliam a eficácia, a eficiência e o impacto de determinados tratamentos; comparam os procedimentos; avaliam custos e benefícios dos serviços prestados; definem estratégias na área da saúde e monitoram a qualidade de vida dos indivíduos, neste caso específico das gestantes.

A utilização de instrumentos tanto genéricos como específicos ajudariam a avaliar as necessidades de saúde que não se restringem aos cuidados biológicos e são articuladas às condiçôes sociais, demográficas e psicológicas da gestante. Protegendo assim os grupos que são considerados mais vulneráveis.

\section{Referências}

1. Lima YMS, Moura MAV. Consulta de enfermagem pré-natal: a qualidade centrada na satisfação da cliente. Revista de Pesquisa: cuidado é fundamental 2005;9(1/2):93-9.

2. Souza JG. Consulta de Enfermagem à gestante: o olhar e o fazer crítico para a resolutividade. [Dissertação]. Universidade Federal do Rio de Janeiro, Rio de Janeiro; 1996.
3. World Health Organization (WHO).The WHOQOL Group. The World Health Organization Quality of Life Assessment (WHOQOL): position paper from the World Health Organization. Social Sci Med 1995;41(10):1403-9.

4. Fayers PMD. Quality of life. Assessment, analysis and interpretation. Chichester: John Wiley; 2000.

5. Rodrigues-Neto JFR, Ferreira GC. Qualidade de vida como medida de desfecho em saúde. Rev Méd Minas Gerais 2003;13(1):42-6.

6. Kude VMM. Como se faz um projeto de pesquisa qualitativa em Psicologia. Psico 1997;28(1):9-34.

7. Aquino NM, Sun SY, Oliveira EM, Martins M da G, Silva JF, Mattar R. Sexual violence and its association with health self-perception among pregnant women. Rev Saúde Pública 2009;43(6):954-60.

8. Mozzambani ACF, Ribeiro R L, Fuso FS, Fiks JP, Mello MF. Gravidade Psicopatológica em mulheres vítimas de violência doméstica. Rev Psiquiatr Rio Gd Sul 2011;33(1):43-7.

9. Lacasse A, Bérard A. Validation of nausea and vomiting of pregnancy specific health related Qol questionnare. Helath Qual Life Outcomes 2008;6:32.

10. Bijlenga D, Boers KE, Birnie E, Mol BW, Vijgen SC, Van der Post JA, et al. Maternal health-related quality of life after induction of labor or expectant monitoring in pregnancy complicated by intrauterine growth retardation beyond 36 weeks. Qual Life Res 2011;20(9):1427-36.

11. Castro DFA. Qualidade de vida de gestantes Assistidas pela Estratégia Saúde da Família [Dissertação]. São Paulo: Escola de Enfermagem, Universidade de São Paulo; 2010.

12. Lima MOP. Qualidade de vida relacionada à saúde de mulheres grávidas com sintomas depressivos. [Tese]. Escola de Enfermagem da USP: São Paulo; 2011.

13. Webster J, Nicholas C, Velacott C, Cridland N, Fawcett L, Aust NZJ. Validation of the WHOQOL-bref among women following childbirth. Obstet Gynaecol 2010;50(2):132-7. 